\title{
Correction to: Optimal Control Involving Sweeping Processes
}

\author{
M. d. R. de Pinho ${ }^{1}$ • M. M. A. Ferreira ${ }^{1}$. G. V. Smirnov ${ }^{2}$
}

Published online: 9 September 2019

(C) Springer Nature B.V. 2019

\section{Correction to: Set-Valued and Variational Analysis 27(2): 523-548 https://doi.org/10.1007/s11228-018-0501-8}

We would like to make corrections to a result, Lemma 2, in the above paper. The main results of this paper remain valid but some changes need to be made. In fact, Lemma 2 is not correctly stated.

We would like to thank Vera Zeidan for alerting us to the fact. The care she put in reading our paper is gratefully acknowledged.

Next we present the corrections needed in the paper.

\section{Section 4: Approximating Control Problem}

The statement of Lemma 2 should read

Lemma 2 Let $(\hat{x}, \hat{u})$ be an optimal process for problem $(P)$. Assume that the hypotheses H1-H6 are satisfied. Consider a sequence $\left\{\gamma_{k}\right\}$ such that

$$
\gamma_{k} \rightarrow+\infty \text { and for all } k, \gamma_{k}>\frac{2 M}{\eta}
$$

The online version of the original article can be found at https://doi.org/10.1007/s11228-018-0501-8.

M. M. A. Ferreira

mmf@fe.up.pt

M. d. R. de Pinho

mrpinho@fe.up.pt

G. V. Smirnov

smirnov@math.uminho.pt

1 Faculdade de Engenharia da Universidade do Porto, DEEC, SYSTEC, Porto, Portugal

2 Centre of Physics, Department of Mathematics and Applications, Universidade do Minho, Braga, Portugal 
and $\alpha$ a non negative real number. For each $k$, there exists $\left\{\left(x_{\gamma_{k}}, u_{\gamma_{k}}\right)\right\}$ that solves

$$
\left(P_{\gamma_{k}}^{\alpha}\right)\left\{\begin{array}{l}
\text { Minimize } \phi(x(1))+\alpha \int_{0}^{1}|u-\hat{u}|^{2} d t+|x(0)-\hat{x}(0)|^{2} \\
\quad+\frac{1}{\sqrt{\gamma_{k}}} \int_{0}^{1}\left|u-u_{\gamma_{k}}\right| d t+\frac{1}{\sqrt{\gamma_{k}}}\left|x(0)-x_{\gamma_{k}}(0)\right| \\
\text { over processes }(x, u) \text { such that } \\
\dot{x}(t)=f(t, x(t), u(t))-\gamma_{k} e^{\gamma_{k} \psi(x(t))} \nabla \psi(x(t)), \quad \text { a.e. } t \in[0,1], \\
u(t) \in U, \quad \text { a.e. } t \in[0,1], \\
x(0) \in C_{0} .
\end{array}\right.
$$

and there exists a subsequence such that

$$
u_{\gamma_{k}}(t) \longrightarrow \hat{u}(t) \text { a.e. and } x_{\gamma_{k}} \longrightarrow \hat{x} \text { uniformly in }[0,1] .
$$

Next we show how the arguments of the proof should change:

Proof The sentence

Under our assumptions, known existence theorems for optimal control problems guarantee that, for each $k$, there exists a solution $\left(x_{\gamma_{k}}, u_{\gamma_{k}}\right)$ for $\left(P_{\gamma_{k}}^{\alpha}\right)$.

should be deleted and replaced by what follows:

Define the set $W$ comprising vectors $e \in C_{0}$ and $L^{2}$ functions $u:[0,1] \rightarrow \mathbb{R}^{m}$ taking values in $U$. Consider the distance in $W$ defined as

$$
d_{W}\left(\left(e_{1}, u_{1}\right),\left(e_{2}, u_{2}\right)\right)=\left|e_{1}-e_{2}\right|+\int_{0}^{1}\left|u_{1}(t)-u_{2}(t)\right| d t .
$$

With respect to this distance the space $W$ is a complete metric space. Moreover, it is a simple matter to see that, under our conditions, the function defined in $W$ by

$$
(e, u) \rightarrow \phi(x(1))+\alpha \int_{0}^{1}|u-\hat{u}|^{2} d t+|x(0)-\hat{x}(0)|^{2},
$$

where $x(1)$ is associated to the solution of

$$
\dot{x}(t)=f(t, x(t), u(t))-\gamma_{k} e^{\gamma_{k} \psi(x(t))} \nabla \psi(x(t)), \quad \text { a.e. } t \in[0,1]
$$

that satisfies $x(0)=e$, is continuous in $W$ and bounded from below. From the Ekeland's variational principle (see [24]) we see that there exists a pair $\left\{\left(x_{\gamma_{k}}, u_{\gamma_{k}}\right)\right\}$ solving $\left(P_{\gamma_{k}}^{\alpha}\right)$.

The remaining of the proof follows with very pointwise changes to accommodate the extra terms

$$
\frac{1}{\sqrt{\gamma_{k}}} \int_{0}^{1}\left|u-u_{\gamma_{k}}\right| d t+\frac{1}{\sqrt{\gamma_{k}}}\left|x(0)-x_{\gamma_{k}}(0)\right|
$$

of the cost function.

In particular, conditions (ii) and (iii) of Proposition 1 as it reads now are:

Proposition 1 Let $\left\{\left(x_{\gamma_{k}}, u_{\gamma_{k}}\right)\right\}$ solve $\left(P_{\gamma_{k}}^{\alpha}\right)$ defined in Lemma 2. Under the conditions of Lemma 2 , there exists an absolutely continuous function $p_{\gamma_{k}}:[0,1] \rightarrow \mathbb{R}^{n}$ such that 
(i) (adjoint equation) ....

(ii) (transversality condition)

$$
\left(p_{\gamma_{k}}(0),-p_{\gamma_{k}}(1)\right) \in\left(2\left(x_{\gamma_{k}}(0)-\hat{x}(0)\right)+\frac{1}{\sqrt{\gamma_{k}}} v_{k}, \partial^{L} \phi\left(x_{\gamma_{k}}(1)\right)\right)+N_{C_{0}}^{L}\left(x_{\gamma_{k}}(0)\right) \times\{0\}
$$

where $v_{k} \in \mathbb{R}^{n}$ and $\left|v_{k}\right|=1$.

(iii) (maximization condition)

$$
\max _{u \in U}\left\{\left\langle f\left(t, x_{\gamma_{k}}, u\right)-\gamma_{k} e^{\gamma_{k} \psi_{\gamma_{k}}} \nabla \psi_{\gamma_{k}}, p_{\gamma_{k}}\right\rangle-\alpha|u-\hat{u}|^{2}-\frac{1}{\sqrt{\gamma_{k}}}\left|u-u_{\gamma_{k}}\right|\right\}
$$

is attained at $u_{\gamma_{k}}(t)$, for almost every $t \in[0,1]$.

Keep in mind that under our assumptions both $\left|\hat{u}-u_{\gamma_{k}}\right|$ and $\left|\hat{x}(0)-x_{\gamma_{k}}(0)\right|$ are bounded and that $\frac{1}{\sqrt{\gamma_{k}}} \rightarrow 0$.

Observe that in Section 6, containing the proof of Theorem 2, some small changes are now called because of the change of statement of Lemma 2. Those are however minor and easy to accommodate and so we refrain from including them.

Publisher's Note Springer Nature remains neutral with regard to jurisdictional claims in published maps and institutional affiliations. 